\title{
Theoretical Modeling of Galactic Cosmic Rays Flux in Solar Cycles: 20 -23 including Delay Time
}

\author{
Marek Siluszyk ${ }^{1}$ \\ Siedlce University \\ Siedlce, Poland \\ E-mail: marek.siluszyk@uph.edu.pl
}

\section{Krzysztof Iskra}

Polish Air Force University

Deblin, Poland

E-mail: k.iskra@law.mil.pl

We analyzed long period changes of Galactic Cosmic Rays (GCR) in different epochs of Solar Magnetic Cycles (SMC); Solar Cycles: 20 -23. The purpose of this paper is to carry on a study of the Delay Time (DT) between the changes of GCR intensity on the one hand and various parameters characterizing conditions in heliosphere, on the other, as tilt angle $\delta$ of the Heliospheric Current Sheet (HCS), module B of the Heliospheric Magnetic Field (HMF) and variations of drift effect of the GCR particles upon Solar Activity (SA). Based on the Parker's Transport Equation (PTE) we expand three dimensional (3-D) time dependent model describing a propagation of the energetic astroparticles (GCR) in heliosphere. The Parker's PTE is a $2^{\text {nd }}$ order partial differential equation contains a well-known four major processes as: diffusion, convection, drift and adiabatic cooling responsible for modulation of the GCR flux in heliosphere. We analyzed long period changes in different epochs of SA. The new idea in present modelling is connected with the using for implementation of two independent parameters as the gamma and nu. The proxies' gamma and nu are calculated from different sources, nu- based on the HMF's hourly data and gamma from neutron monitors (NMs) daily data. The solutions of PTE obtained from numerical models are compared with the changes of the GCR flux measured by NMs. Based on the investigation we obtained DT for the solar cycles: 20 to 23 - an acceptable compatibility is kept when the minimum of the expected temporal changes of the GCR particles density is shifted with respect to the minimum of the temporal changes of the smoothed experimental data of the GCR intensity

36th International Cosmic Ray Conference -ICRC2019-

July 24th - August 1st, 2019

Madison, WI, U.S.A. 


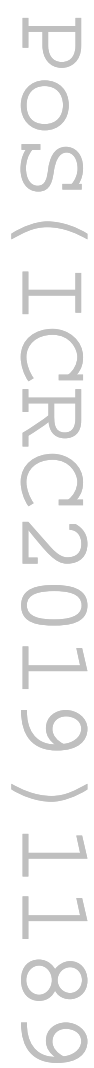

